\title{
Large-magnitude Pelvic and Retroperitoneal Tissue Damage Predicts Organ Failure
}

\author{
Greg Gaski MD, Travis Frantz BS, Scott Steenburg MD, Teresa Bell PhD, \\ Todd McKinley MD
}

Published online: 13 January 2016

(C) The Association of Bone and Joint Surgeons (B) 2016

\begin{abstract}
Background Pelvic and retroperitoneal trauma is a major cause of morbidity and mortality in multiply injured patients. The Injury Severity Score (ISS) has been criticized for underrepresenting and inaccurately defining mechanical injury. The influence of pelvic injury volume on organ dysfunction and multiple organ failure (MOF) has not been described. Through the use of CT, this investigation sought to precisely define volumes of mechanical tissue damage by anatomic region and examine its impact on organ failure.

Questions/purposes (1) Do patients with MOF have a greater volume of pelvic and retroperitoneal tissue damage when compared with those without MOF? (2) In patients

Each author certifies that he or she, or a member of his or her immediate family, has no funding or commercial associations (eg, consultancies, stock ownership, equity interest, patent/licensing arrangements, etc) that might pose a conflict of interest in connection with the submitted article.

All ICMJE Conflict of Interest Forms for authors and Clinical Orthopaedics and Related Research ${ }^{\circledR}$ editors and board members are on file with the publication and can be viewed on request.

Each author certifies that his or her institution approved the human protocol for this investigation, that all investigations were conducted in conformity with ethical principles of research, and that informed consent for participation in the study was obtained.
\end{abstract}

G. Gaski, T. McKinley

Indiana University Health, Methodist Hospital, Department of Orthopaedic Surgery, Indiana University School of Medicine, Indianapolis, IN, USA

T. Frantz

Indiana University School of Medicine, Indianapolis, IN, USA

S. Steenburg

Department of Radiology and Imaging Sciences, Indiana

University School of Medicine, Indianapolis, IN, USA who sustained pelvic trauma, does the magnitude of pelvic injury differ in patients with MOF? (3) Does the magnitude of organ dysfunction correlate with pelvic tissue damage volume?

Methods Seventy-four multiply injured patients aged 18 to 65 years with an ISS $\geq 18$ admitted to the intensive care unit for a minimum of 6 days with complete admission CT scans were analyzed. Each identifiable injury in the head/neck, chest, abdomen, and pelvis underwent volumetric determination using CT to generate regional tissue damage volume scores. Primary outcomes were the development of MOF as measured by the Denver MOF score and the degree of organ dysfunction by utilization of the Sequential Organ Failure Assessment (SOFA) score. Mean pelvic and retroperitoneal tissue damage volumes were compared in patients who developed MOF and those who did not develop MOF using Student's t-test. Among patients who sustained pelvic injuries, we compared mean volume of tissue damaged in patients who developed MOF and those who did not. We assessed whether there was a correlation between organ dysfunction, as measured by the SOFA score as a continuous variable, and the volume of pelvic and retroperitoneal tissue damage using the Pearson product-moment correlation coefficient.

\footnotetext{
T. Bell

Department of Surgery, Center for Outcomes Research in Surgery, Indiana School of Medicine, Indianapolis, IN, USA

G. Gaski ( $\square)$

Indiana University Health, Methodist Hospital, Department of Orthopaedic Surgery, Indiana University School of Medicine, 1801 N Senate Boulevard, Suite 535, Indianapolis, IN 46202, USA

e-mail: ggaski@iuhealth.org; greggaski@gmail.com
} 
Results The average volume of tissue damage was greater in patients with MOF when compared with those without (MOF: $685.667 \pm 1081.344$; non-MOF: $195.511 \pm$ 381.436; mean difference 490.156 cc [95\% confidence interval $\{\mathrm{CI}\}, 50.076-930.237 \mathrm{cc}], \mathrm{p}=0.030)$. Among patients who sustained pelvic injuries, those with MOF had higher average tissue damage volumes than those without MOF (MOF: $1322.000 \pm 1197.050$; non-MOF: $382.750 \pm$ 465.005; mean difference 939.250 [95\% CI, 229.2671649.233], $\mathrm{p}=0.013$ ). Organ dysfunction (SOFA score) correlated with higher volumes of pelvic tissue damage $(\mathrm{r}=$ $0.570, \mathrm{p}<0.001)$.

Conclusions This investigation demonstrated that greater degrees of pelvic and retroperitoneal tissue damage calculated from injury CT scans in multiply injured patients is associated with more severe organ dysfunction and an increased risk of developing MOF. Early identification of polytrauma patients at risk of MOF allows clinicians to implement appropriate resuscitative strategies early in the disease course. Improved stratification of injury severity and a patient's anticipated clinical course may aid in the planning and execution of staged orthopaedic interventions. Future avenues of study should incorporate the ischemic/hypoperfusion component of pelvic injury in conjunction with the mechanical component presented here for improved stratification of multiply injured patients at higher risk of MOF.

Level of Evidence Level III, prognostic study.

\section{Introduction}

Despite advances in resuscitative care of multiply injured patients (MIPs), predicting the clinical trajectory of these patients remains challenging. It is widely known that trauma is the leading cause of death for people younger than 45 years old [3]. For those who survive the initial traumatic insult, the most common cause of death is multiple organ failure (MOF) [6, 13]. Although the incidence of MOF has slightly decreased over the last 15 years, MOF-related complications, intensive care unit (ICU) length of stay (LOS), and mortality have remained relatively constant $[6,22]$. Mechanical tissue damage, ischemic tissue injury (shock), preexisting host factors (age, comorbidities), and the host response all contribute to the development of MOF [24]. MOF prediction models have been described based on age and global physiologic response to injury and resuscitation [24, 29]. The Injury Severity Score (ISS) is the most commonly used instrument to summate regional and whole-body trauma, but this index does not quantify tissue damage volumes.

However, there are limited data to help us understand how the physical components of injury, specifically magnitude and distribution of tissue injury, correspond to the development of organ failure. ISS has been heavily scrutinized for its underrepresentation of multiple severe injuries in one bodily region, underestimation of multiple extremity injury, and its retrospective nature has limited predictive capacity [5, 26]. Reports have implicated head injury, chest injury, and abdominal injury in the etiology of organ dysfunction [17, 24]. Although surgeons have hypothesized that injury magnitude and distribution affect outcomes after injury, mechanical tissue damage has never been precisely quantified in trauma patients. Accurate quantification of mechanical tissue injury, a patient-specific signature of tissue damage, may provide insight toward clinical trajectory and further pathophysiologic understanding of organ dysfunction in trauma patients. Prompt stratification of MIPs at greatest risk to develop MOF and complicated clinical courses may allow early intervention [7]. Pelvic and retroperitoneal trauma has been recognized as a major cause of morbidity and mortality in trauma patients [8]. However, the extent to which pelvic injury corresponds to MOF has, to our knowledge, not been described.

We therefore asked: (1) Do patients with MOF have a greater volume of pelvic and retroperitoneal tissue damage when compared with those without MOF? (2) In patients who sustained pelvic trauma, does the magnitude of pelvic injury differ in patients with MOF? (3) Does the magnitude of organ dysfunction correlate with pelvic tissue damage volume?

\section{Patients and Methods}

\section{Study Design and Setting}

This is an institutional review board-approved retrospective study from a prospectively collected database and medical records of MIPs at a Level I trauma center. All MIPs initially admitted to the ICU or taken to the operating room were identified through querying a trauma database from 2011 to 2012 .

\section{Participants}

Polytrauma patients aged 18 to 65 years with an ISS $\geq 18$ $(n=467)$ admitted to the ICU $(n=353)$ for a minimum of 6 days $(n=81)$ were included in this investigation. All eligible participants were required to have CT of the head/neck, chest, abdomen, and pelvis within 24 hours of presentation (three excluded). Patients with a known history of preexisting organ failure, hematologic disease, immune disorder, and/or currently taking immunomodulating drugs were excluded $(n=4)$. The remaining 74 patients formed the population of interest. 


\section{Description of the Experiment}

Tissue damage was quantified using admission CT scans and imaging software. Each identifiable injury in the head/neck, chest, abdomen, and pelvis underwent volumetric determination using CT. Each injury and surrounding tissue damage had three orthogonal characteristic diameters measured on two orthogonal CT images. Characteristic diameters spanned the region of parenchymal damage and surrounding hematoma. The mean of the three measurements served as the representative diameter of injury. Each injury was assumed to be spherical in nature and the characteristic radius was used to calculate the volume ( $\mathrm{V}=4$ / $3 \pi r^{3}$ ) (Fig. 1). In less than $2 \%$ of injuries, imaging precluded precise measurement in all three dimensions and thus only recordings deemed accurate were used.

Volumes of all injuries were reported as cubic centimeters and summed to generate a total body tissue damage volume score. Tissue damage was categorized by four body regions: (1) head/neck (all injuries superior to the C7/T1 articulation); (2) chest (heart, lungs, chest wall, diaphragm, ribs, thoracic spine, clavicle, and scapula); (3) abdomen (stomach, liver, spleen, gastrointestinal tract to the sigmoid colon, pancreas, abdominal wall); and (4) pelvis/retroperitoneum (pelvis, acetabulum, sacrum, lumbosacral spine, kidneys, urinary bladder, sigmoid colon, rectum, uterus, and testicles). This investigation focused on the impact of pelvic and retroperitoneal tissue damage volumes and the influence of chest, abdomen, and head injury was not explored in this analysis. CT scanning of extremity injuries was inconsistent; therefore, we did not include extremity tissue damage volumes in calculations. Although it can be assumed that $5 \mathrm{cc}$ of intracranial injury will have a larger impact on overall physiology than $5 \mathrm{cc}$ of pelvic hematoma, organ-specific injury analysis was not conducted in this investigation.

Variables, Outcome Measures, Data Sources, and Bias

The primary outcome measures were MOF as defined by the Denver MOF Score and organ dysfunction as measured by the Sequential Organ Functional Assessment (SOFA) score. Both outcome instruments have been validated in a trauma population $[1,9,23]$. MOF was described as a score of $\geq 4$ with involvement of at least two organ systems for the Denver score [23, 24]. The SOFA score evaluates the following six organ systems with an increasing score of dysfunction from 0 to 4 : cardiovascular, respiratory, hepatic, renal, hematologic, and neurologic; the Denver MOF score evaluates the following four organ systems with an increasing score of dysfunction from 0 to 3 : cardiac, pulmonary, hepatic, and renal (Table 1).
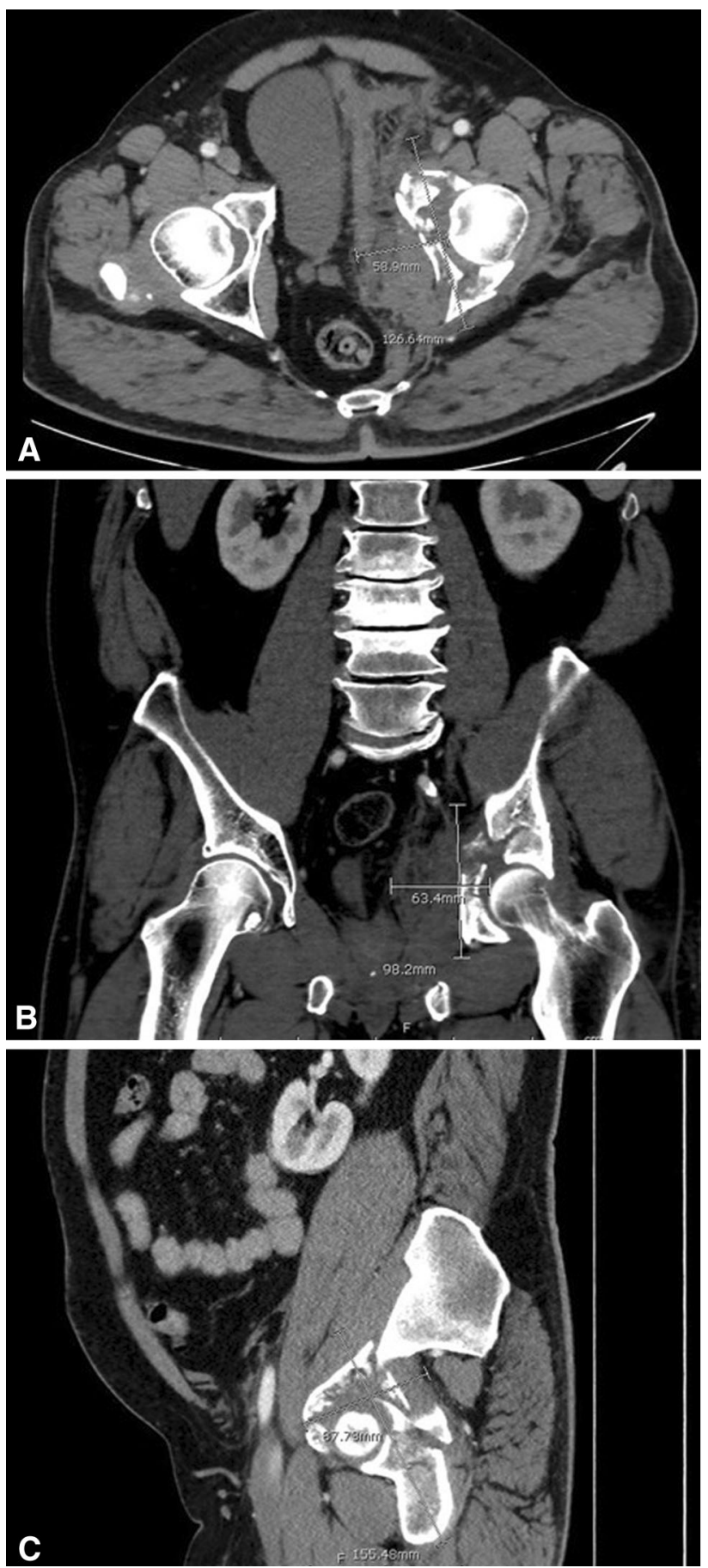

Fig. 1A-C This is an example of CT-based measurements of an acetabular fracture and the surrounding soft tissue injury with characteristic diameters on axial (A), coronal (B), and sagittal (C) cuts.

The SOFA score provides an ongoing measure of organ dysfunction. Higher scores within each organ system correspond with greater degrees of organ dysfunction and SOFA scores were thus measured as a continuous variable. The mean total maximum SOFA score, admission SOFA 


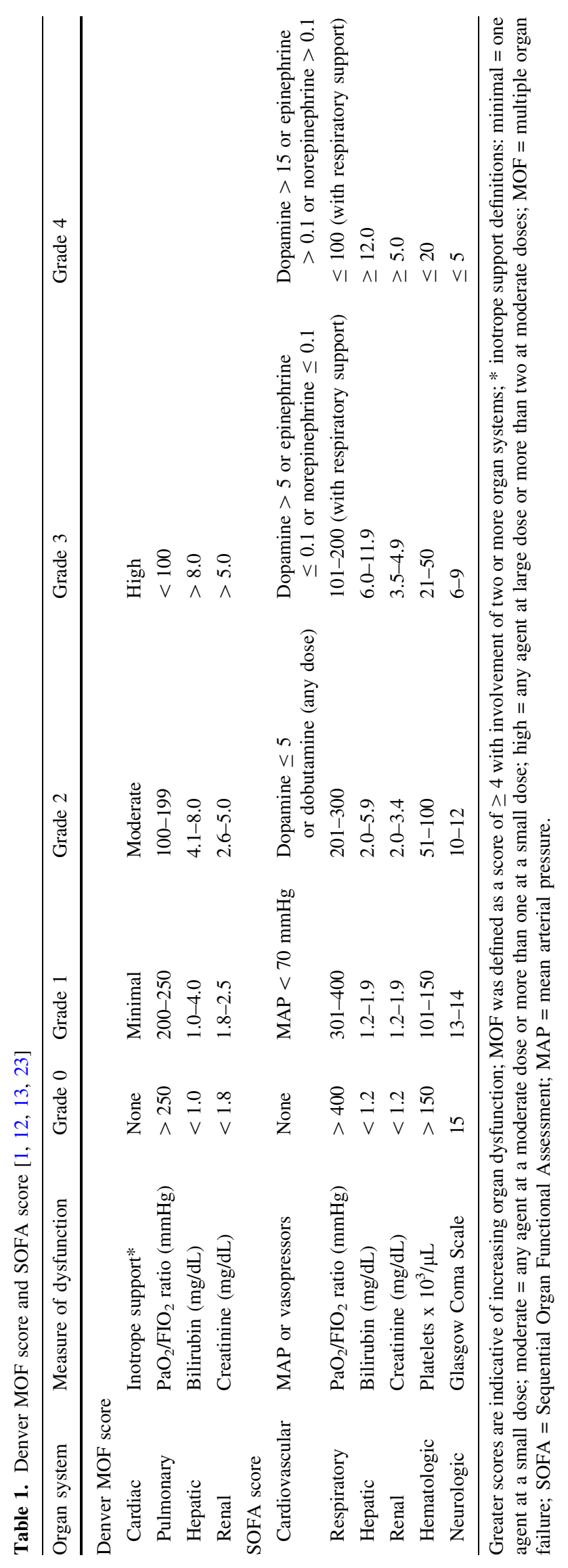


Table 2. Descriptive patient demographics and outcome statistics

\begin{tabular}{lll}
\hline Demographic & Mean & SD \\
\hline Age (years) & 38.92 & 14.01 \\
Sex & $56 \mathrm{M} ; 18 \mathrm{~F}$ & \\
ISS & 31.65 & 9.90 \\
BMI & 30.34 & 7.32 \\
ICU days & 15.81 & 7.72 \\
Death & $6 / 74(8.11 \%)$ &
\end{tabular}

$\mathrm{M}=$ male $; \mathrm{F}=$ female; ISS = Injury Severity Score; BMI = body mass index; ICU $=$ intensive care unit.

score, and delta SOFA (mean total maximum SOFA minus admission SOFA) have all been shown to correlate well with complications and mortality [1, 14]. Comparisons between both widely used outcome instruments for the diagnosis of MOF have demonstrated higher sensitivity with the SOFA score and higher specificity with the Denver MOF score [9].

Neurologic data through Glasgow Coma Scale scores may be inaccurate in therapeutically sedated and obtunded patients and tend to inflate the degree of central nervous system organ failure [9]. For this reason, a modified SOFA score was used in this analysis by excluding the neurologic component.

\section{Statistical Analysis}

Pelvic tissue damage volumes were analyzed as a continuous variable. Descriptive statistics were calculated for all patients. Continuous variables were assessed using oneway analysis of variance or Student's t-test and categorical variables were assessed using chi-square tests. The relationship between mean SOFA scores and volume of pelvic tissue injured was evaluated using Pearson product-moment correlation coefficients. All tests were two-sided and alpha was set at 0.05 .

\section{Demographics}

The cohort was comprised of severely injured polytrauma patients admitted to the ICU for a minimum of 1 week. Demographic data collected included: age, gender, ISS, body mass index, ICU LOS, and mortality (Table 2).

\section{Results}

The volume of pelvic and retroperitoneal tissue damage was greater in patients with MOF when compared with those without (MOF: $685.667 \pm 1081.344$; non-MOF:

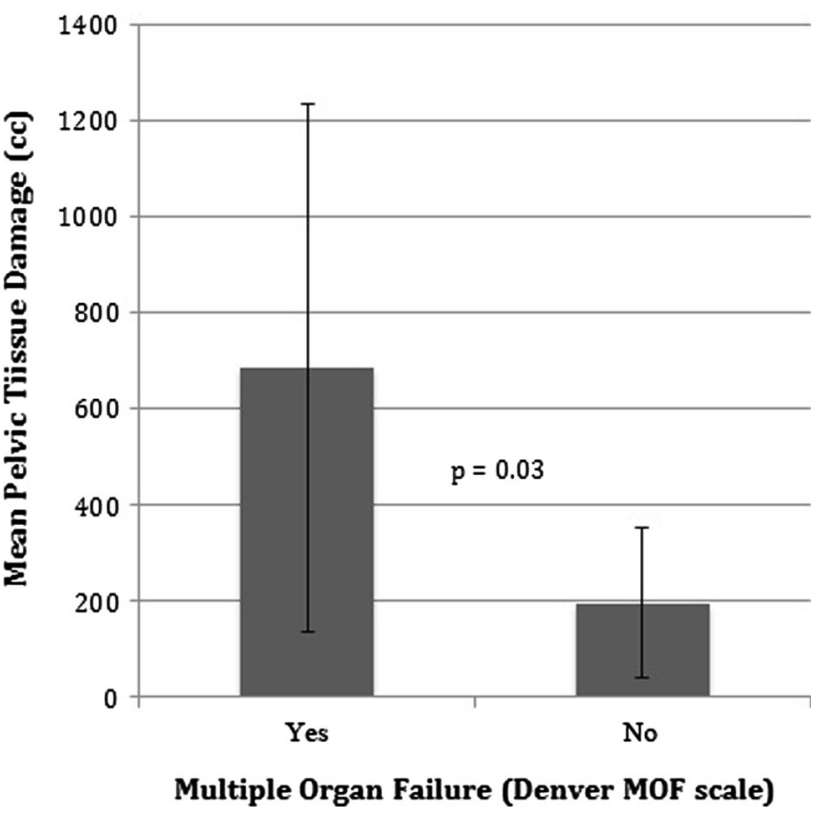

Fig. 2 Patients who developed MOF had higher mean pelvic and retroperitoneal tissue damage volume scores than those who did not succumb to MOF.

$195.511 \pm 381.436 ;$ mean difference 490.156 cc $[95 \%$ confidence interval $\{\mathrm{CI}\}, 50.076-930.237 \mathrm{cc}], \mathrm{p}=0.030$ ) (Fig. 2).

Among patients who sustained pelvic injuries, those with MOF had higher average tissue damage volumes than those without MOF (MOF: $1322.000 \pm 1197.050$; nonMOF: $382.750 \pm 465.005$; mean difference 939.250 [95\% CI, 229.267-1649.233], $\mathrm{p}=0.013$ ).

Larger magnitudes of pelvic tissue damage (cc) were correlated with higher levels of organ dysfunction as measured by the mean modified SOFA score $(r=0.570$, $\mathrm{p}<0.001)$.

\section{Discussion}

MOF is the leading cause of death in MIPs who survive major trauma $[5,12]$. Although clinicians have rightfully focused on correspondence among head, chest, and abdominal injury with MOF, few studies have evaluated the impact and severity of pelvic trauma on the development of MOF [16, 23]. ISS has long been used as a surrogate of mechanical injury; however, it has been shown to consistently underestimate mechanical injury $[4,25]$. Additionally, ISS is a retrospective index and thus inherently limited in its use in prospectively stratifying patient trajectory. We therefore asked the question: Can CT-based imaging provide a more accurate prospective representation of the amount of tissue injured in trauma patients and 
does the volume of injury correlate to patient outcomes? Precise metrics of injury quantification available to the clinician within hours of presentation will allow an enhanced predictive capacity of a patient's clinical course and aid in decision-making such as resuscitative care and resource allocation. This investigation demonstrated that pelvic tissue damage volumes identified patients at risk of MOF and corresponded to severity of organ dysfunction.

This study had a number of limitations. There was potential for selection bias in this study given this cohort represents a severely injured group of patients who were admitted to the ICU for a minimum of 1 week. Regional tissue damage profiles in less severe trauma patients may exhibit different patterns of organ dysfunction and therefore the results presented in this study cannot be extrapolated to a general trauma population. Second, although CT-based injury measurements were derived from multiple axial thin-slice $(2 \mathrm{~mm})$ and reconstructed images, injuries were assumed to be spherical for calculation of individual injury. This methodology likely overestimates individual injury damage volumes because actual tissue damage is not uniformly spherical and a sphere has the greatest volume/surface ratio of any geometric figure. Therefore, the mean values of pelvic tissue damage predictive of MOF and organ dysfunction are likely lower than our calculations. However, all patients had consistent measurement techniques to facilitate between-group comparisons. Additionally, less than $2 \%$ of measurements had injury volumes derived from two reconstructed images instead of three. This was the result of the injury measurement on a third plane being less than $3 \mathrm{~mm}$. Extremity injury analysis was not conducted because most patients with extremity injury did not have a CT scan of the affected limb. Radiographic estimation of injury is considerably inaccurate compared with CT. Although this study focused solely on pelvic and retroperitoneal injury, future investigations of whole-body tissue damage should include methodology to estimate extremity injury.

Our techniques quantified all injury within each region including parenchymal injury and surrounding hematoma. Although hematomas contain inflammatory mediators, the incorporation of hematoma may overestimate the amount of injured tissue. Therefore, larger magnitude pelvic injuries are partly explained by large hematomas.

Patients with MOF have much greater volume of pelvic and retroperitoneal tissue damage, and such damage may predict development of MOF. Previous investigations have focused on the regional impact of head, chest, and/or abdominal trauma on organ dysfunction [7, 10, 17, 22, 24, 27]. These findings parallel previous observations that severity of regional injury may have greater systemic consequences than the mere presence of a regional insult.
Multiple studies have shown that MIPs with axial orthopaedic injuries are at risk of developing systemic complications [2, 15, 18, 19, 28]. Timing and choices of interventions in these patients remain controversial and are largely anecdotal [10-12, 16, 20, 21, 27]. Surgeons readily agree that invasive operations must be carefully staged in MIPs at risk for organ dysfunction. However, judging which patients are at elevated risk of organ dysfunction is typically elusive. The findings of this study indicate that patients sustaining greater degrees of pelvic and retroperitoneal tissue damage are more likely to develop MOF and higher levels of organ dysfunction. This risk can be numerically stratified from routine admission CT. Understanding these risks can guide surgical decisions for staged orthopaedic interventions in MIPs.

Greater levels of pelvic and retroperitoneal tissue damage are positively correlated with higher levels of organ dysfunction. Previous studies have largely focused on the association of pelvic injury with mortality [4, 25]. For example, in 30,000 trauma patients of whom over 1000 had a pelvic injury, Schulman et al. [25] found the presence of a pelvic ring injury to be an independent risk factor for mortality after controlling for other systemic injury. In contrast, the effect of pelvic and retroperitoneal injury on the development of organ failure has not been explored. Our results revealed increasing volumes of pelvic tissue damage were correlated with higher levels of organ dysfunction in this population of critically injured patients.

Patients with pelvic injuries are known to have an increased risk of mortality [4, 25]. Data from this investigation showed larger magnitude pelvic injuries correlate with higher levels of organ dysfunction and predicted MOF. Early identification of polytrauma patients at risk of MOF permits patient-specific injury stratification and institution of early treatment strategies. The timing and magnitude of subsequent orthopaedic interventions may be more effectively implemented with an enhanced understanding of a patient's risk of developing organ failure and a complicated clinical course. Future research should focus on refinement of the volumetric quantification methods used here to more precisely define the injury burden in a prospective fashion.

Acknowledgments We thank our research coordinators, Krista Brown and Lauren Hill, for their assistance in multiple aspects of this investigation.

\section{References}

1. Antonelli M, Moreno R, Vincent JL. Application of SOFA score to trauma patients. Intensive Care Med. 1999;25:389-394.

2. Bliemel C, Lefering R, Buecking B, Frink M, Struewer J, Krueger A, Rucholtz S, Frangen TM. Early or delayed 
stabilization in severely injured patients with spinal fractures? Current surgical objectivity according to the Trauma Registry of DGU: treatment of spine injuries in polytrauma patients. $J$ Trauma Acute Care Surg. 2014;76:366-373.

3. Burgess AR, Eastridge BJ, Young JW, Ellison TS, Ellison PS Jr, Poka A, Bathon GH, Brumback RJ. Pelvic ring disruptions: effective classification system and treatment protocols. J Trauma. 1990;30:848-856.

4. Centers for Disease Control and Prevention. 2015. Available at: http://www.cdc.gov/injury/wisqars/leadingcauses.html. Accessed March 2015.

5. Chawda MN, Hildebrand F, Pape HC, Giannoudis PV. Predicting outcome after multiple trauma: which scoring system? Injury Int J Care Injured. 2004;35:347-358.

6. Ciesla DJ, Moore EE, Johnson JL, Burch JM, Cothren CC, Sauaia A. A 12-year prospective study of postinjury multiple organ failure: has anything changed? Arch Surg. 2005;140:432-438.

7. Cryer HG, Leong K, McArthur DL, Demetriades D, Bongard FS, Fleming AW, Hiatt JR, Kraus JF. Multiple organ failure: by the time you predict it, it's already there. J Trauma. 1999;46:597-604.

8. Cullinane DC, Schiller HJ, Zielinski MD, Bilaniuk JW, Collier BR, Como J, Holevar M, Sabater EA, Sems SA, Vassy WM, Wynne JL. Eastern Association for the Surgery of Trauma practice management guidelines for hemorrhage in pelvic fractureupdate and systematic review. J Trauma. 2011;71:1850-1868.

9. Dewar DC, White A, Attia J, Tarrant SM, King KL, Balogh ZJ. Comparison of postinjury multiple-organ failure scoring systems: Denver versus Sequential Organ Failure Assessment. J Trauma Acute Care Surg. 2014;77:624-629.

10. Flierl MA, Stoneback JW, Beauchamp KM, Hak DJ, Morgan SJ, Smith WR, Stahel PF. Femur shaft fixation in head-injured patients: when is the right time? J Orthop Trauma. 2010;24:107114.

11. Jaicks RR, Cohn SM, Moller BA. Early fracture fixation may be deleterious after head injury. J Trauma. 1997;42:1-5.

12. Lefaivre KA, Starr AJ, Stahel PF, Elliott AC, Smith WR. Prediction of pulmonary morbidity and mortality in patients with femur fracture. $J$ Trauma. 2010;69:1527-1535.

13. Moore FA, Sauaia A, Moore EE, Haenel JB, Burch JM, Lezotte DC. Postinjury multiple organ failure: a bimodal phenomenon. $J$ Trauma. 1996;40:501-512.

14. Moreno F, Vincent JL, Matos R, Mendonca A, Cantraine F, Thijs L, Takala J, Sprung C, Antonelli M, Bruining H, Willatts S. The use of maximum SOFA score to quantify organ dysfunction/failure in intensive care. Results of a prospective, multicentre study. Intensive Care Med. 1999;25:686-696.

15. Morshed A, Miclau T $3^{\text {rd }}$, Bembom O, Cohen M, Knudson MM, Colford JM Jr. Delayed internal fixation of femoral shaft fracture reduces mortality among patients with multisystem trauma. $J$ Bone Joint Surg Am. 2009;91:3-13.

16. Nahm NJ, Como JJ, Wilber JH, Vallier HA. Early appropriate care: definitive stabilization of femoral fractures within 24 hours of injury is safe in most patients with multiple injuries. J Trauma. 2011;71:175-185.

17. Nast-Kolb D, Aufmkolk M, Rucholtz S, Obertacke U, Waydhas C. Multiple organ failure still a major cause of morbidity but not mortality in blunt multiple trauma. J Trauma. 2001;51:835-841.

18. O'Toole RV, O’Brien M, Scalea TM, Habashi N, Pollak AN, Turen $\mathrm{CH}$. Resuscitation before stabilization of femoral fractures limits acute respiratory distress syndrome in patients with multiple traumatic injuries despite low use of damage control orthopedics. J Trauma. 2009;67:1013-1021.

19. Pape HC, Giannoudis PV, Krettek C, Trentz O. Timing of fixation of major fractures in blunt polytrauma. Role of conventional indicators in clinical decision making. $J$ Orthop Trauma. 2005; 19:551-562.

20. Pape HC, Hildebrand F, Pertschy S, Zelle B, Garapati R, Grimme $\mathrm{K}$, Krettek C, Reed RL 2nd. Changes in the management of femoral shaft fractures in polytrauma patients: from early total care to damage control orthopaedic surgery. $J$ Trauma. 2002;53:452-462.

21. Probst C, Probst T, Ganesslen A, Krettek C, Pape HC. Timing and duration of the initial pelvic stabilization after multiple trauma in patients from the German Trauma Registry: is there an influence on outcome? J Trauma. 2007;62:370-377.

22. Sauaia A, Moore EE, Johnson JL, Chin TL, Banerjee A, Sperry JL, Maier RV. Temporal trends of postinjury multiple organ failure: still resource intensive, morbid, and lethal. J Trauma Acute Care Surg. 2014;76:582-593.

23. Sauaia A, Moore EE, Johnson JL, Ciesla DJ, Biffl WL, Banerjee A. Validation of postinjury multiple organ failure scores. Shock. 2009;31:438-447.

24. Sauaia A, Moore FA, Moore EE, Norris JM, Lezotte DC, Hamman RF. Multiple organ failure can be predicted as early as 12 hours after injury. J Trauma. 1998;45:291-303.

25. Schulman JE, O'Toole RV, Castillo RC, Manson T, Sciadini MF, Whitney A, Pollak AN, Nascone JW. Pelvic ring fractures are an independent risk factor for death after blunt trauma. J Trauma. 2010;68:930-934.

26. Shin E, Evans KN, Fleming ME. Injury score underpredicts injury severity and resource utilization in combat-related amputations. J Orthop Trauma. 2013;27:419-423.

27. Townsend RN, Lheureau T, Protetch J, Riemer B, Simon D. Timing of fracture repair in patients with severe brain injury (Glasgow Coma Scale score). J Trauma Acute Care Surg. 1998;44:977-983.

28. Vallier HA, Wang X, Moore TA. Timing of orthopaedic surgery in multiple trauma patients: development of a protocol for early appropriate care. J Orthop Trauma. 2013;27:543-551.

29. Vogel JA, Liao MM, Hopkins E, Seleno N, Byyny RL, Moore EE, Gravitz C, Haukoos JS. Prediction of postinjury multipleorgan failure in the emergency department: development of the Denver Emergency Department Trauma Organ Failure Score. $J$ Trauma Acute Care Surg. 2014;76:140-145. 